\title{
CORRELATION OF TYPE OF SPECIES AND PARASITE DENSITY IN MALARIA WITH PLATELET COUNT, MEAN PLATELET VOLUME AND PLATELET DISTRIBUTION WIDTH
}

\author{
Nidhish Kumar ${ }^{1}$ \\ ${ }_{1}^{1}$ Postgraduate Student, Department of Pathology, Kasturba Medical College, Mangalore.
}

\section{ABSTRACT}

\section{BACKGROUND}

Malaria is an important infectious protozoan disease and it has been estimated that worldwide there are 300 - 500 million cases of malaria per year and 1.5 - 2.7 million deaths. The early diagnosis of malaria is the key feature for its prompt treatment and prevention of complications. The study was conducted to statistically analyse other haematological parameters like platelet indices that is Mean Platelet Volume (MPV) and Platelet Distribution Width (PDW) along with platelet count. Correlation of the above was done with parasite density and type of species. An attempt was made to study whether platelet indices and counts are indicative of type of infection.

\section{MATERIALS AND METHODS}

The study was conducted prospectively over a period of one month in the Haematology Laboratory of Kasturba Medical College Hospital, Mangalore. It included cases of acute malaria which presented with fever within a week caused either by Plasmodium vivax (PV), falciparum (PF) or dual infection (PV + PF). All cases were diagnosed by demonstration of the respective parasite by Quantitative Buffy Coat assay. Haematological parameters which included platelet count (PC), mean platelet volume (MPV) and platelet distribution width (PDW) were recorded and statistically analysed.

\section{RESULTS}

The study included 100 cases of malarial infection with maximum cases in the age group of 10-30 years. Out of 100 cases 86 were PV positive, 6 cases were PF positive and 8 cases of mixed infection. From above graphs, there was no correlation between falciparum parasitaemia with either PDW $(\mathrm{P}=0.58)$ or MPV $(\mathrm{P}=0.16)$, similar to vivax infection. The drop in platelet count with increasing parasitaemia of vivax schizonts was highly significant $(\mathrm{P}<0.001)$, but not falciparum infection $(\mathrm{P}=0.00)$. The increase in $\mathrm{PDW}$ was significant with increasing MPV $(\mathrm{P}=0.003)$ and simultaneous decrease in platelet count $(\mathrm{P}=0.00)$.

\section{CONCLUSION}

Increased vivax parasitaemia, unlike falciparum parasitaemia, was significantly associated with thrombocytopaenia. The increase in PDW was significantly associated with increase in MPV and simultaneous decrease in platelet count. Correlation of parasitaemia in either of malaria species with changes in MPV and PDW, was not significant.

\section{KEYWORDS}

Plasmodium Vivax (PV), Plasmodium Falciparum (PF), Platelet Count (PC), Mean Platelet Volume (MPV), Platelet Distribution Width (PDW).

HOW TO CITE THIS ARTICLE: Kumar N. Correlation of type of species and parasite density in malaria with platelet count, mean platelet volume and platelet distribution width. J. Evolution Med. Dent. Sci. 2016;5(89):6622-6625, DOI: 10.14260/Jemds/2016/1498

\section{BACKGROUND}

Malaria is a Protozoal disease caused by infection with parasites of the genus Plasmodium (Pl).(1) They are transmitted to humans by certain species of female Anopheline mosquito, which are infected.(1) Five species of Plasmodium (Plasmodium Vivax, Plasmodium Falciparum, Plasmodium Ovale, Plasmodium Malariae and Plasmodium Knowlesi) cause malaria in humans.(1) An estimated 243 million cases of malaria were present in 2008, of which $85 \%$ were in African region followed by South-East Asia region

Financial or Other, Competing Interest: None.

Submission 02-10-2016, Peer Review 25-10-2016,

Acceptance 02-11-2016, Published 05-11-2016.

Corresponding Author:

Dr. Nidhish Kumar,

C/o. Dr. Mohan Singh,

Jagat Narayan Road,

Kadam Kuan, Patna-800003,

Bihar.

E-mail: nidhishsingh7@gmail.com

DOI: $10.14260 /$ jemds $/ 2016 / 1498$
(10\%) and 4\% were East Mediterranean.(1) Approximately, 863,000 people died of malaria of which $89 \%$ were from African region.(1) Malaria is an important infectious protozoan disease and early diagnosis of malaria is a key feature of its prompt treatment and prevention of complications.(2) Main complications of malaria are coma, hypoglycaemia, acidosis, renal failure and pulmonary oedema.(2) Microscopic diagnosis is needed for confirmation of malaria, but it requires technical expertise and may be at times unreliable when poorly executed.(3) Haematological changes which include low platelet count, haemoglobin concentration and haematocrit have been reported to be associated with malaria.(4,5) Many recently conducted studies have shown the role of platelet indices to determine the various causes of malaria and thrombocytopaenia.(6) This study was conducted to statistically analyse other haematological parameters like Mean Platelet Volume (MPV), Platelet Distribution Width (PDW) along with the platelet count. Correlation of the above was done with parasite density and type of species. An attempt was made to study whether platelet indices and counts are indicative of type of infection. 


\section{MATERIALS AND METHODS}

The present study was conducted prospectively over a period of one month in the Department of Pathology, Kasturba Medical College, Mangalore, a premier tertiary care centre in coastal Karnataka. Cases were collected from Manipal Hospital Jyothi Circle and Manipal Hospital Attavar. Sample size of 100 cases was taken. The study included cases of acute malaria which presented with fever within a week caused either by Plasmodium Vivax (PV), Plasmodium Falciparum (PF) or dual infection (PV $+\mathrm{PF}$ ). All the cases were diagnosed by demonstration of the respective parasite by Quantitative Buffy Coat Assay. Haematological parameters which included Platelet Count (PC), Mean Platelet Volume (MPV) and Platelet Distribution Width (PDW) was recorded. The automated analyser used was Beckman coulter LH780. Quantitative Buffy Coat (QBC) was done using QBC centrifuge and QBC capillary blood tube at 12000 rotations per minute for 5 minutes using Acridine Orange stain to highlight the parasite. The fluorescent microscope used was Olympus Cx21i. The parasites were viewed under $\mathrm{QBC}$ immersion oil with a magnification of $60 \mathrm{x}$ at objective and $10 \mathrm{x}$ at eye piece. The parasites were graded as $1+$ when parasite density was less than 1 per High Power Field (HPF), 2+ when parasite density was between 1 - 10 per HPF, $3+$ when between $10-100$ per HPF and 4+ when more than 1000 per HPF. Statistical analysis was done using ANOVA (Fisher's test). P value of $<0.001$ was found to be statistically significant.

\section{RESULTS}

Hundred cases which were proved positive by QBC technique were selected. Out of 100 cases 90 were PV schizonts positive, 64 were PV trophozoites positive and 18 were PF trophozoites positive. Age range for selected cases was 1 to 79 years with mean being 34.7. Platelet count, PDW and MPV was noted. Platelet count range was 9000 to 305,000 with mean being 114,700 . Similarly, PDW range was 15.6 to 19.4 with mean being 17.4 and MPV range being 6.1 to 11.6 with mean being 8.91. Schizonts of PV were seen in 90 out of 100 cases of which 29 were $1+, 25$ were $2+, 18$ were $3+$ and 18 were $4+$. PV trophozoites were seen in 64 cases out of which 11 were $1+$, 20 were $2+, 21$ were $3+$ and 12 were $4+$. PF trophozoites were seen in 18 cases out of which 8 were $1+, 4$ were $2+$ and 6 were $3+$.

\begin{tabular}{|c|c|c|}
\hline & Frequency & Percent \\
\hline$<10$ & 3 & 3.0 \\
\hline $10-20$ & 16 & 16.0 \\
\hline $20-30$ & 35 & 35.0 \\
\hline $30-40$ & 15 & 15.0 \\
\hline $40-50$ & 11 & 11.0 \\
\hline $50-60$ & 10 & 10.0 \\
\hline$>60$ & 10 & 10.0 \\
\hline Total & $\mathbf{1 0 0}$ & $\mathbf{1 0 0 . 0}$ \\
\hline \multicolumn{3}{|c|}{ Table 1. Age Group Distribution } \\
\hline
\end{tabular}

\begin{tabular}{|c|c|c|c|c|c|c|}
\hline & & $\mathbf{N}$ & Mean & Std. Deviation & $\mathbf{F}$ & p \\
\hline \multirow{4}{*}{ pdw } & $1+$ & 29 & 17.2966 & .87606 & & \\
\hline & $2+$ & 25 & 17.3120 & .82579 & & \\
\hline & $3+$ & 18 & 17.6889 & .92666 & & \\
\hline & $4+$ & 18 & 17.6278 & .63599 & 1.33 & .27 \\
\hline \multirow{4}{*}{$\mathrm{mpv}$} & $1+$ & 29 & 8.4345 & 1.12449 & & \\
\hline & $2+$ & 25 & 9.0760 & 1.32893 & & \\
\hline & $3+$ & 18 & 9.2056 & 1.10638 & & \\
\hline & $4+$ & 18 & 9.2278 & .94918 & 2.72 & .05 \\
\hline \multirow{5}{*}{ platelet } & $1+$ & 29 & 152068.9655 & 74523.21356 & & \\
\hline & $2+$ & 25 & 109080.0000 & 65485.95269 & & \\
\hline & $3+$ & 18 & 96777.7778 & 48093.72658 & & \\
\hline & $4+$ & 18 & 75000.0000 & 41621.54420 & & \\
\hline & Total & & & & 6.60 & $<0.001$ VHS \\
\hline
\end{tabular}

There was no correlation between parasitaemia of vivax schizont with either PDW $(\mathrm{P}=0.27)$ or MPV $(\mathrm{P}=0.05)$. There was no correlation between parasitaemia of vivax trophozoites with either PDW $(\mathrm{P}=0.83)$ or MPV $(\mathrm{P}=0.71)$.

There was no correlation between parasitaemia of falciparum with either PDW $(\mathrm{P}=0.58)$ or MPV $(\mathrm{P}=0.16)$. However, drop in platelet count with increasing parasitaemia of vivax schizonts was highly significant $(\mathrm{P}<0.001)$.

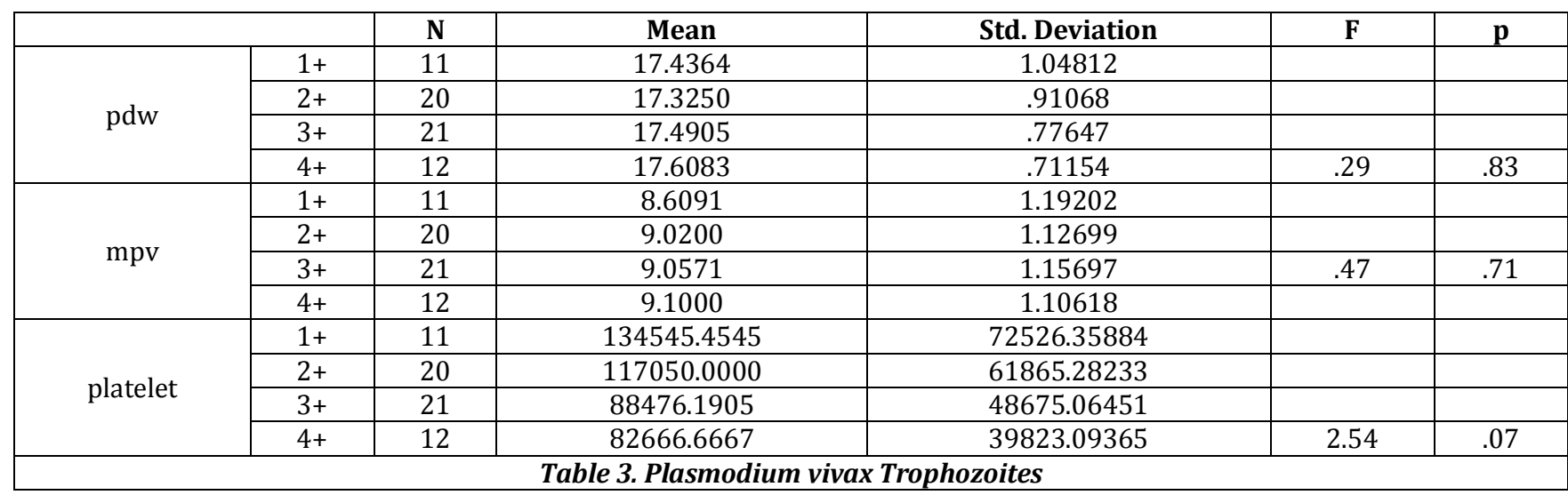




\begin{tabular}{|c|c|c|c|c|c|c|}
\hline & & $\mathbf{N}$ & Mean & Std. Deviation & $\mathbf{F}$ & $\mathbf{p}$ \\
\hline \multirow{3}{*}{ pdw } & $1+$ & 8 & 17.1750 & .68400 & & \\
\hline & $2+$ & 4 & 17.2750 & .63966 & & \\
\hline & $3+$ & 6 & 16.8000 & .98387 & .56 & .58 \\
\hline \multirow{3}{*}{ mpv } & $1+$ & 8 & 9.0375 & .89752 & & \\
\hline & $2+$ & 4 & 9.7750 & .60208 & & \\
\hline & $3+$ & 6 & 8.6833 & .86583 & 2.07 & .16 \\
\hline \multirow{3}{*}{ platelet } & $1+$ & 8 & 133875.0000 & 64368.22086 & & \\
\hline & $2+$ & 4 & 61500.0000 & 30005.55504 & & \\
\hline & $3+$ & 6 & 115666.6667 & 74518.90141 & 1.78 & .20 \\
\hline \multicolumn{7}{|c|}{ Table 4. Plasmodium falci Trophozoites } \\
\hline
\end{tabular}

\begin{tabular}{|c|c|c|c|}
\hline \multicolumn{2}{|c|}{} & MPV & Platelet \\
\hline \multirow{3}{*}{$\mathrm{pdw}$} & $\mathrm{R}$ & .291 & -.543 \\
\cline { 2 - 4 } & $\mathrm{P}$ & .003 & .000 \\
\cline { 2 - 4 } & $\mathrm{N}$ & 100 & 100 \\
\hline \multirow{3}{*}{$\mathrm{mpv}$} & $\mathrm{R}$ & & -.500 \\
\cline { 2 - 4 } & $\mathrm{P}$ & & .000 \\
\cline { 2 - 4 } & $\mathrm{N}$ & 100 \\
\hline \multicolumn{3}{|c|}{ Table 5. Correlations } \\
\hline
\end{tabular}

The increase in PDW was significant with increasing MPV $(\mathrm{P}=0.003)$ and simultaneous decrease in platelet count $(\mathrm{P}=0.000)$.

\begin{tabular}{|c|c|c|c|c|}
\hline \multicolumn{3}{|c|}{ Sex } & MPV & Platelet \\
\hline \multirow{6}{*}{ Male } & \multirow{3}{*}{$\mathrm{pdw}$} & $\mathrm{R}$ & .343 & -.514 \\
\hline & & $\mathrm{P}$ & .004 & .000 \\
\hline & & $\mathrm{N}$ & 69 & 69 \\
\hline & \multirow{3}{*}{$\mathrm{mpv}$} & $\mathrm{R}$ & & -.515 \\
\hline & & $\mathrm{P}$ & & .000 \\
\hline & & $\mathrm{N}$ & & 69 \\
\hline \multirow{6}{*}{ Female } & \multirow{3}{*}{$\mathrm{pdw}$} & $\mathrm{R}$ & .315 & -.655 \\
\hline & & $\mathrm{P}$ & .085 & .000 \\
\hline & & $\mathrm{N}$ & 31 & 31 \\
\hline & \multirow{3}{*}{ mpv } & $\mathrm{R}$ & & -.480 \\
\hline & & $\mathrm{P}$ & & .006 \\
\hline & & $\mathrm{N}$ & & 31 \\
\hline \multicolumn{5}{|c|}{ Table 6. Correlations } \\
\hline
\end{tabular}

\section{DISCUSSION}

Malaria is caused by $\mathrm{Pl}$. vivax and $\mathrm{Pl}$. falciparum, which is endemic in many parts of India.(1) Malaria is a true haematological disease, which affects almost all blood components.(1) Most frequently malaria associated haematological complications are thrombocytopaenia and anaemia.(1) Malaria has been reported as the major cause of low platelet counts in the endemic areas. (1) In some places, it is used as an indicator of malaria in patients presenting with fever as a characteristic feature. Platelets count of less than $150,000 /$ cumm increases the chance of malaria 12-15 times. $(7,8,9)$

$\mathrm{Pl}$. vivax trophozoite infection was most common in our study with a positivity of $64 \%$. Pl. falciparum trophozoites were seen in $18 \%$ of the cases. A similar observation was made by Gupta et al, which showed $56.51 \%$ positivity for Pl. Vivax and $39.13 \%$ positivity for Pl. falciparum infection. Similar results were seen in a study done by Faseela et al.(10)

In our study, increase in parasitaemia of $\mathrm{Pl}$. vivax was highly significant with the drop in platelet count with $\mathrm{p}$ value being $<0.001$. In a study done by Gupta et al, thrombocytopaenia was seen in $78 \%$ of cases.(1) Similar observations were made by Colonel et al, which showed thrombocytopaenia in $72 \%$ of cases.(11) A study done by Jamal et al on paediatric patients reported thrombocytopaenia in $72 \%$ of patients with malaria infection.(12)

Exact mechanism of thrombocytopaenia is still unknown in malaria. Fajardo and Tallent demonstrated a direct lytic effect of the parasite on platelets and also Pl. vivax within platelets.(13)

Both non-immunological destruction as well as immune mechanism(14) involving specific platelet associated IgG antibodies that bind directly to malarial antigen in the platelets have been recently reported to play a role in the lysis of platelets.(15)

Oxidative stress damage of platelets has also been implicated in the aetiopathogenesis based on the finding of low levels of platelet superoxide-dismutase and glutathione peroxidase activity and high platelet lipid peroxidation levels in malaria patients when compared to those of healthy subjects.(16)

Decreased thrombopoiesis has been ruled out, because platelet forming megakaryocytes in the marrow are usually normal or increased.(16) A good tolerance of low platelet count is well-known in malaria.

In our study, Pl. vivax malaria was found to have more significant thrombocytopaenia. More cases of thrombocytopaenia in vivax malaria infection may attribute to a possible development of a new genotype of Pl. vivax.(16) Recent studies have shown that thrombocytopaenia is equally or even more common in Pl. vivax malaria contrary to the popular belief that it may be observed in $\mathrm{Pl}$. falciparum malaria.(17)

It is recently seen in India that thrombocytopaenia exhibited a heightened frequency and severity among patients with Pl. vivax infections.(18) Recent studies conducted from Indian subcontinent have found significant thrombocytopaenia in Pl. vivax malaria.(19,20) Studies from Qatar and Venezuela had shown the similar results.(21,22) There is no matched control group. This is one of the limitations of this study.

\section{CONCLUSION}

Higher frequency of mild-to-severe thrombocytopaenia is observed in patients suffering from malaria. The above finding can have therapeutic implications in context of avoiding unnecessary platelet infusion in malaria patients. Presence of thrombocytopaenia in a patient with acute febrile illness in the tropics increases the possibility of malaria. This may be used in addition to the clinical and microscopic parameters to heighten the suspicion of this disease and prompt initiation of the treatment. 


\section{REFERENCES}

1. Gupta NK, Bansal SB, Jain UC, et al. Study of thrombocytopenia in patients of malaria. Tropical Parasitology 2013;3(1):58-61.

2. Chandra S, Chandra H. Role of haematological parameters as an indicator of acute malarial infection in Uttarakhand state of India. Mediterranean Journal of Hematology and Infectious Diseases 2013;5(1):e2013009.

3. World Health Organisation. New Perspectives, malaria diagnosis. (Geneva) 2000.

http://www.who.int/malaria/publications/atoz/who_cds _rbm_2000_14/en/index.html PMid:21757937.

4. Igbeneghu $\mathrm{C}$, Odaibo $\mathrm{AB}$, Olaleye DO. Impact of asymptomatic malaria on some hematological parameters in the Iwo community in Southwestern Nigeria. Med Princ Pract 2011;20(5):459-63.

5. Kumar A, Shashirekha. Thrombocytopenia--an indicator of acute vivax malaria. Indian $\mathrm{J}$ Pathol Microbiol 2006;49(4):505-8.

6. Chandra H, Chandra S, Rawat A, et al. Role of mean platelet volume as discriminating guide for bone marrow disease in patients with thrombocytopenia. Int J Lab Hematol 2010;32(5):498-505.

7. Maina RN, Walsh D, Gaddy C, et al. Impact of plasmodium falciparum infection on haematological parameters in children living in Western Kenya. Malar J 2010;9 (Suppl3):S4.

8. Adedapo AD, Falade CO, Kotila RT, et al. Age as a risk factor for thrombocytopenia and anaemia in children treated for acute uncomplicated falciparum malaria. J Vector Borne Dis 2007;44(4):266-71.

9. Lathia TB, Joshi R. Can hematological parameters discriminate malaria from nonmalarious acute febrile illness in the tropics? Indian J Med Sci 2004;58(6):239-44.

10. Faseela TS, Roche, Anita KB, et al. Diagnostic value of platelet count in malaria. J Clin Diagn Res 2011;5:464-6.

11. Colonel KM, Bhika RD, Khalid S, et al. Severe thrombocytopenia and prolonged bleeding time in patients with malaria (a clinical study of 162 malaria cases). World Appl Sci J 2010;9(5):484-8.
12. Jamal A, Memon IA, Latif F. The association of plasmodium vivax malaria with thrombocytopenia in febrile children. Pak Paediatr J 2007;31(2):85-9.

13. Fajardo LF. Malarial parasites within human platelets. J Am Med Assoc 1974;229(9):1205-7.

14. Jadhav UM, Patkar VS, Kadam NN. Thrombocytopenia in malaria: correlation with type and severity of malaria. J Assoc Physicians India 2004;52:615-8.

15. Makkar RP, Mukhopadhyay S, Monga A, et al. Plasmodium vivax malaria presenting with severe thrombocytopenia. Braz J Infect Dis 2002;6(5):263-5.

16. Metanat M, Sharifi-Mood B. Malaria vivax and severe thrombocytopenia in Iran. Iran J Parasitol 2010;5(3):69-70.

17. Aggarwal A, Rath S, Shashiraj. Plasmodium vivax malaria presenting with severe thrombocytopenia. J Trop Pediatr 2005;51(2):120-1.

18. Kochar DK, Das A, Kochar A, et al. Thrombocytopenia in plasmodium falciparum, plasmodium vivax and mixed infection malaria: a study from bikaner (Northwestern India). Platelets 2010;21(8):623-7.

19. Srivastava S, Ahmad S, Shirazi N, et al. Retrospective analysis of vivax malaria patients presenting to tertiary referral centre of Uttarakhand. Acta Trop 2011;117(2): 8-5.

20. George P, Alexander LM. A study on the clinical profile of complicated plasmodium vivax mono-infections. Asian Pac J Trop Med 2010;3:560-2.

21. Khan FY, Lutof AK, Yassin MA, et al. Imported malaria in qatar: a one year hospital-based study in 2005. Travel Med Infect Dis 2009;7(2):111-7.

22. González B, Rodulfo H, De Donato $M$, et al. Hematologic variations in patient with malaria caused by plasmodium vivax before, during and after treatment. Invest Clin 2009;50(2):187-201. 